\title{
Genetic diversity of the Algerian peanut population analyzed using morphological markers and seed storage proteins
}

\author{
DOI: $10.30901 / 2227-8834-2021-3-111-124$ \\ УДК 633.852.52:631.526.32:631.527(65) \\ Поступление/Received: 18.03.2021 \\ Принято/Accepted: 02.09.2021
}

\section{H. DJEGHIM ${ }^{1}$, I. BELLIL ${ }^{2}$, D. KHELIFI ${ }^{3}$}

${ }^{1}$ National Center for Biotechnology Research,

P.O. Box E73, Nouvelle Ville Ali Mendjeli, Constantine 25016, Algeria

- livenanou@hotmail.com

${ }^{2}$ Frères Mentouri University,

P.O. Box 325, Ain El Bey Way, Constantine 25017, Algeria

bines07@yahoo.fr

${ }^{3}$ National School of Biotechnology,

P.O. Box E66, Ville Universitaire Ali Mendjeli, Constantine

25100, Algeria

dkhelifi@yahoo.fr
Генетическое разнообразие

алжирской популяции арахиса, изученной с использованием морфологических маркеров

и запасных белков семян

\section{Х. ДЖЕГХИМ ${ }^{1}$, И. БЕЛЛИЛЬ², Д. КХЕЛИФИ}

${ }^{1}$ Национальный центр биотехнологических исследований, 25016 Алжир, г. Константин, Нувель Вилль Али Менджели, $n / я$ Е73

livenanou@hotmail.com

${ }^{2}$ Университет Фрэр Ментури, 25017 Алжир, г. Константин, пр. Айн Эль Бей, n/я 325 bines07@yahoo.fr

${ }^{3}$ Национальная высшая школа биотехнологии, 25100 Алжир, г. Константин, Вилль Юниверситэр Али Менджели, $n / я$ Е66 هdkhelifi@yahoo.fr
Background. The peanut is one of the most important oil crops suitable for cultivation in the tropical areas of the world. Despite its agronomic importance, few studies have been carried out to assess the morphogenetic diversity of Arachis hypogaea L., especially in East African countries. The major interest of this morphologic study lies in the potential of this species to provide useful genes for the improvement of cultivated peanuts. To date, no study has been performed in Algeria to characterize local peanut varieties. Materials and methods. Thirty peanut accessions were collected from four principal areas of peanut production in Algeria. Genetic characterization using 15 agronomic characters and 25 morphological descriptors showed a high level of diversity among accessions. Principal Component Analysis and the Hierarchical Ascendant Classification were made to clarify the genetic relationship between peanut accessions.

Results and discussion. Results showed that leaflet size (length and width), seed shape and size, oil content, and branching pattern were the principal characters to discriminate the screened A. hypogaea accessions. In addition to that, the weights of 10 pods and 100 seeds were the most variable traits and presented a CV of $42.53 \%$ and $40.12 \%$, respectively. On the other hand, total storage proteins extracted were separated using SDS-PAGE and revealed thirty bands that were used to generate a matrix and make a cluster analysis using the UPGMA method, exhibiting different storage proteins compositions. Moreover, the phenotypic diversity observed agrees with the storage protein profile diversity, while the accessions grouped in similar clusters belong to the two subspecies of $A$. hypogaea. The results of the current study show that morphological traits and seed storage proteins can be useful for exploring the diversity among $A$. hypogaea accessions.

Key words: Arachis hypogaea, phenotypic diversity, Principal Component Analysis, SDS-PAGE.
Актуальность. Арахис - одна из важнейших масличных культур, пригодных для возделывания в тропических зонах мира. Несмотря на его хозяйственную ценность, были предприняты лишь редкие попытки изучить и оценить морфогенетическое разнообразие Arachis hypogaea L., особенно в странах Восточной Африки. Основная цель настоящего морфологического исследования - изучить потенциал данного вида как источника ценных генов для улучшения культурных форм арахиса. До сегодняшнего дня в Алжире не проводились исследования по выявлению характеристик местных сортов арахиса.

Материалы и методы. Тридцать образцов арахиса собрали в четырех основных районах его производства в Алжире. Генетическая характеристика с использованием 15 агрономических и 25 морфологических признаков показала высокий уровень разнообразия среди образцов. Для выяснения генетической взаимосвязи между образцами арахиса провели кластерный анализ и анализ главных компонент.

Результаты и обсуждение. Показано, что размер листочков (длина и ширина), форма и размер семян, содержание масла и характер ветвления были основными признаками, позволяющими различить отобранные образцы Arachis hypogaea L. В дополнение к этому, массы 10 бобов и 100 семян были наиболее изменчивыми характеристиками и имели $\mathrm{CV} 42,53 \%$ и 40,12\% соответственно. Был проведен анализ запасных белков семян. Все они были разделены с использованием метода SDS-PAGE, который выявил тридцать полос. Последние были использованы для создания матрицы и кластерного анализа методом UPGMA и продемонстрировали различия образцов арахиса по составу запасных белков. Наблюдаемое фенотипическое разнообразие согласуется с разнообразием профиля запасных белков, и группы образцов в сходных кластерах принадлежали двум подвидам $A$. hypogaea. Результаты данного исследования показывают, что морфологические признаки и запасные белки семян могут быть полезны для изучения разнообразия образцов арахиса.

Ключевые слова: Arachis hypogaea, фенотипическое разнообразие, анализ главных компонент, SDS-PAGE. 


\section{Introduction}

Peanut, or groundnut (Arachis hypogaea L.), is an important oilseed crop cultivated worldwide. In 2018, 45.9 million tons were produced over an area of 28.5 million hectares (FAOSTAT, 2020). A. hypogaea can play an important role in sustainable agricultural development, particularly where drought and salinity frequently limit crop production in the Maghreb and the sub-Saharan regions (Giuffrè et al., 2016). Peanut cultivars have a very narrow genetic base, and there is a lack of information about their morphological and agricultural characteristics. Accurate information on genetic diversity helps the plant breeders in choosing the diverse parents for focused hybridization (Kushwah et al., 2016). Additionally, analysis of storage proteins is considered one of the most powerful methods for describing plant species and lines (Liang et al., 2006). Thus, the aim of the present study was to characterize the peanut accessions currently cultivated in the four main growing areas in Algeria. The specific objectives of the investigation were to estimate phenotypic diversity for different morphological descriptors and agronomic characters, reveal the importance of these traits in addition to their interrelationships, and assess the seed storage protein diversity in peanut for identification of accessions.

\section{Material and methods}

Thirty peanut (A. hypogaea) accessions were collected from different peanut growing areas in Algeria (Fig. 1), including El Taref, Ghardaïa, El Oued, and Adrar (Table 1). Seedlings were grown and maintained under greenhouse conditions at the National Center for Biotechnology Research in Constantine, Algeria $\left(36^{\circ} 24.75^{\prime} \mathrm{N}, 6^{\circ} 57.05^{\prime} \mathrm{E}\right.$, and $773 \mathrm{~m}$ above sea level). During their growth, plants were irrigated as needed. Finally, seeds were collected manually, air-dried in the dark, and stored in paper bags at $4^{\circ} \mathrm{C}$.

\section{Plant characters measured}

Two selected categories of characters from the IBPGR/ ICRISAT peanut (groundnut) descriptors (Descriptors for Groundnut, 1992) were studied: qualitative (morphological) and quantitative (agronomic) characters. They were scored with two to fifteen states and were analyzed. These parameters were measured in three replicates per accession (Table 2).

\subsection{Oil content}

To measure oil content in peanut accessions, oil was extracted using the Soxhlet apparatus (FOSS, SoxtecTM 8000), for each $1 \mathrm{~g}$ of kernel peanut placed on the thimbles, $25 \mathrm{ml}$ of hexane was poured on the extraction vessels. The difference of vessel weight before and after extraction showed oil content. The oil content of each accession was measured in triplicate and results were expressed as the percentage of total oil content.

\subsection{Storage protein extraction}

Storage proteins were extracted from single crushed seeds according to U. K. Laemmli's (1970) protocol with minor modifications. After removing the skin, the peanut kernels were finely milled and defatted with hexane $(10 \mathrm{ml} / \mathrm{g}$ dry weight) at $-20^{\circ} \mathrm{C}$ overnight. $100 \mathrm{mg}$ of dry peanut powder was mixed with $100 \mu \mathrm{l}$ of fresh extraction solution, the mixture was incubated at $65^{\circ} \mathrm{C}$ for $30 \mathrm{~min}$ and then centrifuged $1 \mathrm{~min}$ at $10,000 \mathrm{~g}$.

\section{Data analysis}

Quantitative morphological data were first subjected to descriptive analysis and ANOVA tests and the correlation matrix was performed on all quantitative characters to determine the relationships between them. The frequency table was used for a comparison of means by the analysis of variance (ANOVA) using Tukey's test $(p<0.05)$. The structure of morphological diversity was described using Principal Component Analysis (PCA), and the Hierarchical Ascendant Classification (CAH) was used to explore the relationship between

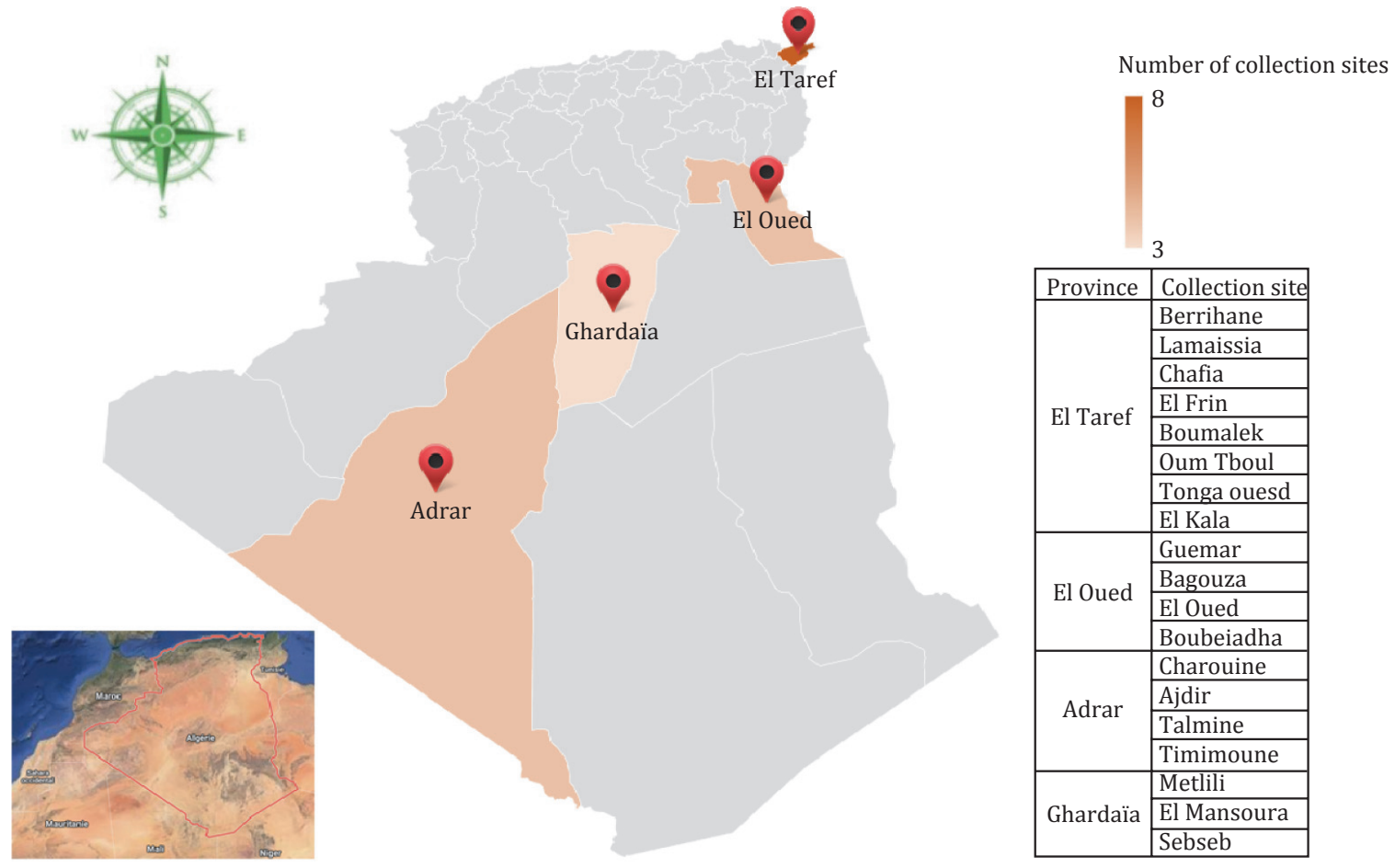

Fig. 1. Map of sampling locations for peanut accessions

Рис. 1. Карта мест сбора образцов арахиса 
Table 1. Geographic origin of the studied Algerian peanut (Arachis hypogaea L.) accessions Таблица 1. Географическое происхождение изученных алжирских образцов арахиса (Arachis hypogaea L.)

\begin{tabular}{|c|c|c|c|c|}
\hline $\begin{array}{l}\text { Geographic } \\
\text { origin }\end{array}$ & $\begin{array}{c}\text { Region where } \\
\text { collected / location }\end{array}$ & Accessions name & $\begin{array}{l}\text { Geographic } \\
\text { zone }\end{array}$ & $\begin{array}{l}\text { Bioclimatic } \\
\text { floor }\end{array}$ \\
\hline \multirow{14}{*}{ Taref } & \multirow{2}{*}{ Berrihane, El Kala } & Berrihane 1 & \multirow{14}{*}{ Coastal } & \multirow{14}{*}{ Subhumid } \\
\hline & & Berrihane 2 & & \\
\hline & \multirow{2}{*}{ Lamaissia, El Kala } & Lamaissia 1 & & \\
\hline & & Lamaissia 2 & & \\
\hline & \multirow{2}{*}{ Chefia, El Kala } & Chefia 1 & & \\
\hline & & Chefia 2 & & \\
\hline & \multirow{4}{*}{ El Kala } & El Kala 1 & & \\
\hline & & El Kala 2 & & \\
\hline & & El Kala 3 & & \\
\hline & & El Kala 4 & & \\
\hline & El Frin, El Kala & El Frin & & \\
\hline & Boumalek, El Kala & Boumalek & & \\
\hline & Oum Tboul, El Kala & Oum Tboul & & \\
\hline & Tonga-Ouest, El Kala & Tonga-Ouest & & \\
\hline \multirow{5}{*}{ Ghardaïa } & \multirow{3}{*}{ Sebseb } & Sebseb 1 & \multirow{11}{*}{ Northern Sahara } & \multirow{16}{*}{ Saharan } \\
\hline & & Sebseb 2 & & \\
\hline & & Sebseb 3 & & \\
\hline & El Mansoura & El Mansoura & & \\
\hline & Metlili & Metlili & & \\
\hline \multirow{6}{*}{ El Oued } & \multirow{2}{*}{ Boubeiadha, Taghzout } & Boubeiadha 1 & & \\
\hline & & Boubeiadha 2 & & \\
\hline & Guemar & El Oued 1 & & \\
\hline & Bagouza, Taghzout & El Oued 2 & & \\
\hline & \multirow{2}{*}{ El Oued } & El Oued 3 & & \\
\hline & & El Oued 4 & & \\
\hline \multirow{5}{*}{ Adrar } & \multirow{2}{*}{ Charouine } & Hiba 1 & \multirow{5}{*}{ Central Sahara } & \\
\hline & & Hiba 2 & & \\
\hline & Ajdir, Timimoune & Adrar 1 & & \\
\hline & Talmine, Timimoune & Adrar 2 & & \\
\hline & Talmine, Timimoune & Timimoune & & \\
\hline
\end{tabular}


Table 2. Morphological and agronomic descriptors used for statistical analysis

Таблица 2. Морфологические и хозяйственно ценные признаки, использованные в статистическом анализе

\begin{tabular}{|c|c|c|c|c|c|}
\hline & $\begin{array}{l}\text { Abbrevi- } \\
\text { ation }\end{array}$ & Character name & Character state & $\begin{array}{c}\text { Range - Frequency (\%) } \\
\text { Average }\end{array}$ \\
\hline \multirow{20}{*}{ 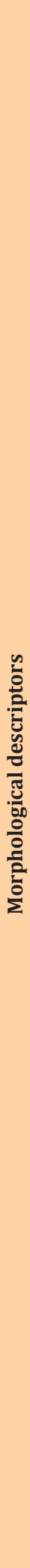 } & \multirow{6}{*}{ के } & Gr.hab & Growth habit & $\begin{array}{l}0 \text { - Erect; } 1 \text { - Decumbent- } 1 \\
2 \text { - Decumbent-2; } 3 \text { - Decumbent-3; } \\
4 \text { - Procumbent- } 2\end{array}$ & $\begin{array}{l}0-46.67 ; 1-10.00 \\
2-3.33 ; 3-36.67 \\
4-3.33\end{array}$ \\
\hline & & Bra.pat & Branching pattern & $\begin{array}{l}0 \text { - Irregular without flowers on main stem; } \\
1 \text { - Irregular with flowers on main stem; } \\
2 \text { - Alternate; } 3 \text { - Sequential }\end{array}$ & $\begin{array}{l}0-3.33 ; 1-23.33 \\
2-13.33 ; 3-40.00\end{array}$ \\
\hline & & Num.bra & Number of branches & 0 - Secondary; 1 - Tertiary & $0-63.33 ; 1-36.67$ \\
\hline & & St.pig & Stem pigmentation & 0 - Absent; 1 - Present & $0-50.00 ; 1-50.00$ \\
\hline & & St.src & Stem surface & $\begin{array}{l}0 \text { - Glabrous; } 1 \text { - Sub-glabrous, hairs in } \\
\text { one or two rows along the main stem; } \\
2 \text { - Moderately hairy, three or four rows } \\
\text { along the main stem; } 3 \text { - Very hairy, most of } \\
\text { the stem surface covered with hairs; } \\
4 \text { - Woolly }\end{array}$ & $\begin{array}{l}0-30.00 ; 1-40.00 \\
2-6.67 ; 3-0.00 \\
4-3.33\end{array}$ \\
\hline & & Bch.dty & Branching density & 0 - Moderate; 1 - High & $0-20.00 ; 1-80.00$ \\
\hline & \multirow{4}{*}{$\frac{\bar{d}}{\frac{1}{1}}$} & Inf.typ & Inflorescence type & 0 - Simple; 1 - Complex & $0-26.67 ; 1-73.33$ \\
\hline & & Peg.pig & Peg pigmentation & 0 - Absent; 1 - Present & $0-56.67 ; 1-43.33$ \\
\hline & & Std.pet.col & Standard petal color & 0 - Yellow 9B; 1 - Orange 24A & $0-66.67 ; 1-33.33$ \\
\hline & & Flo.dis & Flower distribution & $\begin{array}{l}0 \text { - Alternate; } 1 \text { - Complex; } \\
2 \text { - Continuous }\end{array}$ & $\begin{array}{l}0-33.33 ; 1-20.00 \\
2-46.67\end{array}$ \\
\hline & \multirow{5}{*}{ 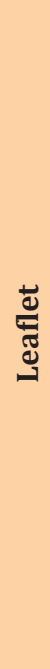 } & Lflt.shp & Leaflet shape & $\begin{array}{l}0 \text { - Oblong-lanceolate; } 1 \text { - Oblong; } \\
2 \text { - Obovate; } 3 \text { - Ovate; } 4 \text { - Suborbicular; } \\
5 \text { - Wide-elliptic; } 6 \text { - Narrow-elliptic; } \\
7 \text { - Oblong-elliptic; } 8 \text { - Elliptic; } \\
9 \text { - Cuneate }\end{array}$ & $\begin{array}{l}0-3.33 ; 1-6.67 \\
2-23.33 ; 3-6.67 \\
4-3.33 ; 5-6.67 \\
6-16.67 ; 7-13.33 \\
8-6.67 ; 9-13.33\end{array}$ \\
\hline & & Lflt.src & Leaflet surface & $\begin{array}{l}0 \text { - Almost glabrous on both surfaces; } \\
1 \text { - Almost glabrous above, hairs below; } \\
2 \text { - Almost glabrous above, hairs and/or } \\
\text { bristles below }\end{array}$ & $\begin{array}{l}0-70.00 ; 1-20.00 \\
2-10.00\end{array}$ \\
\hline & & Lflt.mgn & Leaflet margin & 0 - Entire; 1 - Hairy & $0-40.00 ; 1-60.00$ \\
\hline & & Lflt.tip & Leaflet tip & 0 - Obtuse; 1 - Acute & $0-56.67 ; 1-43.33$ \\
\hline & & Lef.col & Leaf color & $\begin{array}{l}0 \text { - Green 129; } 1 \text { - Green 134A; } \\
2 \text { - Green 135; } 3 \text { - Green 140B; } \\
4 \text { - Green 142; } 5 \text { - Yellow-green 144C; } \\
6 \text { - Yellow-green } 149\end{array}$ & $\begin{array}{l}0-3.33 ; 1-13.33 \\
2-6.67 ; 3-13.33 \\
4-20.00 ; 5-26.67 \\
6-16.67\end{array}$ \\
\hline & $\ddot{\circ}$ & Nod.cap & Nodulation capacity & 0 - Without nodules; 1 - With nodules & $0-80.00 ; 1-20.00$ \\
\hline & \multirow{4}{*}{ : } & Pod.csn & Pod constriction & $\begin{array}{l}0 \text { - None; } 1 \text { - Slight; } 2 \text { - Moderate; } \\
3 \text { - Deep; } 4 \text { - Very deep }\end{array}$ & $\begin{array}{l}0-40.00 ; 1-13.33 \\
2-20.00 ; 3-20.00 \\
4-6.67\end{array}$ \\
\hline & & Txt.pod.src & $\begin{array}{l}\text { Texture of } \\
\text { pod surface }\end{array}$ & 0 - Fine; 1 - Medium; 2 - Coarse & $\begin{array}{l}0-20.00 ; 1 \text { - 13.33; } \\
2-66.67\end{array}$ \\
\hline & & Pod.bk & Pod beak & $\begin{array}{l}0 \text { - Absent; } 1 \text { - Slight; } 2 \text { - Moderate; } \\
3 \text { - Prominent; } 4 \text { - Very prominent }\end{array}$ & $\begin{array}{l}0-13.33 ; 1-20.00 \\
2-50.00 ; 3-3.33 \\
4-13.33\end{array}$ \\
\hline & & Pod.ret & Pod reticulation & $\begin{array}{l}0 \text { - None; } 1 \text { - Slight; } 2 \text { - Moderate; } \\
3 \text { - Prominent; } 4 \text { - Very prominent }\end{array}$ & $\begin{array}{l}0-10.00 ; 1-36.67 \\
2-3.33 ; 3-23.33 \\
4-26.67\end{array}$ \\
\hline
\end{tabular}


Table 2. The end

Таблица 2. Окончание

\begin{tabular}{|c|c|c|c|c|c|}
\hline & & $\begin{array}{l}\text { Abbrevi- } \\
\text { ation }\end{array}$ & Character name & Character state & $\begin{array}{c}\text { Range - Frequency }(\%) \\
\text { Average }\end{array}$ \\
\hline & & Num.sd.pod & $\begin{array}{l}\text { Number of seeds } \\
\text { per pod }\end{array}$ & $0-2-1 ; 1-2-3-1 / 2-1-3 ; 2-3-2-1 / 3-1-2$ & $\begin{array}{l}0-73.33 ; 1-16.67 \\
2-10.00\end{array}$ \\
\hline & & Teg.asp & Tegument aspect & 0 - One color; 1 - Variegated & $0-56.67 ; 1-43.33$ \\
\hline$\frac{n}{2}$ & & Sd.shp & Seed shape & $\begin{array}{l}0 \text { - Round; } 1 \text { - Ovate; } 2 \text { - Oblong; } \\
3 \text { - Cylindric }\end{array}$ & $\begin{array}{l}0-10.00 ; 1-33.33 \\
2-33.33 ; 3-23.33\end{array}$ \\
\hline $\begin{array}{l}\frac{0}{0} \\
\frac{0}{0} \\
\frac{0}{00} \\
\frac{0}{0} \\
\frac{0}{2} \\
\frac{0}{2}\end{array}$ & ¿ूँ & Teg.col & Tegument color & $\begin{array}{l}0 \text { - Orange-yellow 6; } 1 \text { - Orange-yel- } \\
\text { low } 19 ; 2 \text { - Orange } 26 ; 3 \text { - Orange } 28 ; \\
4 \text { - Orange 29; } 5 \text { - Red-orange } 31 ; \\
6 \text { - Red-orange } 33 ; 7 \text { - Red-orange } 34 ; \\
8 \text { - Red-orange } 35 ; 9 \text { - Pink-red 37; } \\
10 \text { - Grey-orange } 165 ; 11 \text { - Grey-oran- } \\
\text { ge } 172 ; 12 \text { - Grey-orange } 175 ; 13 \text { - Grey- } \\
\text { orange } 177 ; 14 \text { - Grey-orange } 178\end{array}$ & $\begin{array}{l}0-3.33 ; 1-6.67 ; \\
2-16.67 ; 3-3.33 \\
4-3.33 ; 5-6.67 \\
6-3.33 ; 7-10.00 \\
8-3.33 ; 9-3.33 \\
10-3.33 ; 11-6.67 \\
12-10.00 ; 13-13.33 \\
14-6.67\end{array}$ \\
\hline & & Sd.col & Seed color & $\begin{array}{l}0 \text { - Yellow } 1 ; 1 \text { - Yellow } 4 ; 2 \text { - Yellow } 5 ; \\
3 \text { - Yellow } 8 ; 4 \text { - Yellow } 10 ; 5 \text { - Yellow } 13\end{array}$ & $\begin{array}{l}0-3.33 ; 1-63.33 \\
2-3.33 ; 3-13.33 \\
4-10.00 ; 5-6.67\end{array}$ \\
\hline & & Pl.wdth & $\begin{array}{l}\text { Plant width or } \\
\text { Spread }(\mathrm{cm})\end{array}$ & Between 06 to 17 & 11.00 \\
\hline & $\stackrel{\Xi}{\infty}$ & Hght.stm & $\begin{array}{l}\text { Height of main stem } \\
(\mathrm{cm})\end{array}$ & Between 28 to 61 & 33.00 \\
\hline & & Dys.mty & Days to maturity & $\begin{array}{l}0 \text { - Between } 80 \text { to } 100 \text { days; } \\
1 \text { - Between } 100 \text { to } 120 \text { days; } \\
2 \text { - Between } 120 \text { to } 140 \text { days }\end{array}$ & $\begin{array}{l}0-56.67 ; 1-26.67 \\
2-16.67\end{array}$ \\
\hline & $\begin{array}{l}\dot{0} \\
\vdots \\
\frac{0}{1}\end{array}$ & Dys.50\%.fwg & $\begin{array}{l}\text { Days to } 50 \% \text { flower- } \\
\text { ing }\end{array}$ & $\begin{array}{l}0 \text { - Between } 20 \text { and } 45 \text { days; } \\
1 \text { - Between } 45 \text { and } 75 \text { days }\end{array}$ & $0-70.00 ; 1-30.00$ \\
\hline$\cong$ & & Lflt.lgth & Leaflet length (mm) & Between 19.08 to 59.35 & 40.27 \\
\hline$\stackrel{2}{\bar{Z}}$ & 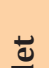 & Lflt.wdth & Leaflet width (mm) & Between 10.44 to 31.45 & 21.01 \\
\hline تِ & త్త & Lef.are & Leaf area $\left(\mathrm{cm}^{2}\right)$ & Between 4.29 to 11.22 & 6.93 \\
\hline อุ & & Chlo.ctn & $\begin{array}{l}\text { Chlorophyll content } \\
\text { (SPAD unit) }\end{array}$ & Between 27.40 to 49.9 & 22.50 \\
\hline & & Pod.lgth & Pod length (mm) & Between 10.26 to 55.79 & 45.53 \\
\hline & $\overline{0}$ & Pod.wdth & Pod width (mm) & Between 5.62 to 20.13 & 14.51 \\
\hline & & $\begin{array}{l}\text { Wght.10 } \\
\text { pods }\end{array}$ & $\begin{array}{l}\text { Weight of } 10 \text { pods } \\
\text { (gr) }\end{array}$ & Between 5.32 to 20.92 & 15.6 \\
\hline & & Wght.100sd & $\begin{array}{l}\text { Weight of } 100 \text { seeds } \\
\text { (gr) }\end{array}$ & Between 30.37 to 108.4 & 78.03 \\
\hline & จุ & Sd.lgth & Seed length $(\mathrm{mm})$ & Between 8.5 to 22.32 & 13.79 \\
\hline & & Sd.wdth & Seed width (mm) & Between 6.23 to 12.26 & 6.03 \\
\hline & & Oil.ctn & Oil content (\%) & Between 36.68 to 56.17 & 19.49 \\
\hline
\end{tabular}


the peanut accessions using arithmetic averages method (UPGMA); these analyses were carried out using data analysis software (XLSTAT 2016.02).

Concerning data analysis of storage proteins, electrophoresis profiles were analyzed using the Image Lab software version 5.1 (Biorad). The presence/absence of a band was scored as $1 / 0$, respectively, and the employment of the Euclidean distance to the binary matrix served to calculate the pairwise distance among the accessions, which were clustered by the UPGMA method.

\section{Results}

\section{Variation in morphological and agronomic charac-} ters

The frequency of appearance of morphological descriptors in the studied 30 accessions of $A$. hypogaea is presented in Table 2. The analysis of variance (ANOVA) showed a high significant variation $(p<0.001)$ among the peanut accessions for all of 13 agronomical characters assessed. Table 3 discloses a descriptive statistics estimation of all characters. The most variable ones included the weights of 10 pods and 100 seeds that ranged from $5.32 \mathrm{~g}$ to $20.92 \mathrm{~g}$ with a CV of $42.53 \%$, and from $30.37 \mathrm{~g}$ to $108.4 \mathrm{~g}$ with a CV of $40.12 \%$, respectively. These results can be attributed to the important variation observed in seed and pod sizes.

\section{Relationships between agronomic characters}

Pearson's correlation coefficients revealed significant associations among the evaluated different agronomic characters. Generally, stem architecture characters were significantly correlated with seed and pod characters. The highest significant correlation coefficients were noticed between leaflet length / leaflet width and the weight of 10 pods / seed width ( $r=0.751, p<0.0001$, and $r=0.776, p<0.0001$, respectively). Furthermore, seed length and pod length were positively and significantly correlated $(r=0.767, p<0.0001)$, hence any restriction of pod growth may cause smaller seeds. These results corroborated those reported by M. A. Zaman et al. (2011). A significant but weak correlation was also observed between leaflet length / plant width ( $\mathrm{r}=0.456, p<0.0001)$, oil content / 10 pod weight $(\mathrm{r}=0.330, p<0.0001)$, and between the height of the main stem / pod width ( $\mathrm{r}=0.302, p<0.0001)$ (see Table 3). Among leaf characters, leaf length was highly and significantly correlated with leaflet surface $(\mathrm{r}=0.553$, $p<0.0001$ ), but did not correlate with leaf area. On the other hand, branching pattern was inversely correlated with seed length and the weight of 10 pods $(\mathrm{r}=-0.594, p<0.0001$, and $r=-0.554, p<0.0001$, respectively). Slight correlations were observed between plant width / leaflet shape and growth habit / nodulation capacity $(\mathrm{r}=0.488, p<0.0001$, and $r=0.324, p<0.0001$, respectively).

Table 3. Descriptive statistics estimation of agronomical characters assessed in the studied accessions

Таблица 3. Описательно-статистическая оценка хозяйственно ценных признаков у изучаемых образцов

\begin{tabular}{|l|c|c|c|c|c|c|}
\hline $\begin{array}{c}\text { Quantitative } \\
\text { character }\end{array}$ & Min & Max & Mean \pm SE & CV\% & $p$ & $\begin{array}{c}\text { Fisher effect } \\
\text { size, \% }\end{array}$ \\
\hline Hght.stm & 28.00 & 61.00 & $44.93 \pm 7.77$ & 17.30 & $<0.0001$ & 13.32 \\
\hline Pl.wdth & 6.00 & 17.00 & $9.69 \pm 2.62$ & 26.98 & $<0.0001$ & 3.10 \\
\hline Lflt.lgth & 19.08 & 59.35 & $42.27 \pm 8.21$ & 19.43 & $<0.0001$ & 4.36 \\
\hline Lflt.wdth & 10.44 & 31.45 & $21.88 \pm 4.21$ & 19.23 & $<0.0001$ & 10.46 \\
\hline Chlo.ctn & 27.40 & 49.90 & $39.48 \pm 5.23$ & 13.25 & $<0.0001$ & 2.36 \\
\hline Lef.are & 4.29 & 11.22 & $6.58 \pm 1.39$ & 21.10 & $<0.001$ & 7.80 \\
\hline Pod.lgth & 10.26 & 55.79 & $28.39 \pm 8.89$ & 31.32 & $<0.0001$ & 16.52 \\
\hline Pod.wdth & 5.62 & 20.13 & $13.41 \pm 3.06$ & 22.84 & $<0.0001$ & 1.30 \\
\hline Wght.10 pod & 5.32 & 20.92 & $11.98 \pm 5.09$ & 42.53 & $<0.0001$ & 11.68 \\
\hline Wght.100 sd & 30.37 & 108.40 & $53.05 \pm 21.29$ & 40.12 & $<0.0001$ & 4.75 \\
\hline Sd.lgth & 8.53 & 22.32 & $14.74 \pm 3.71$ & 25.15 & $<0.0001$ & 9.17 \\
\hline Sd.wdth & 6.23 & 12.26 & $8.59 \pm 1.38$ & 16.06 & $<0.0001$ & 1.91 \\
\hline Oil.ctn & 36.68 & 56.17 & $45.02 \pm 4.01$ & 8.90 & $<0.0001$ & 13.29 \\
\hline
\end{tabular}

Note: $\mathbf{S E}$ - standard error, $\mathbf{C V} \%$ - coefficient of variation, $\boldsymbol{p}$ - probability value

Примечание: SE - стандартная ошибка, CV\% - коэффициент вариации, $\boldsymbol{p}$ - уровень значимости

On the other hand, leaflet width and length revealed similar coefficients of variation among the studied accessions (19.23\% and $19.43 \%$, respectively) with an important positive correlation confirmed by the principal component analysis (Fig. 2, a). However, these characters; in addition to chlorophyll content, presented the least coefficients of variation (8.90\% and $16.06 \%$, respectively).

\section{Principal Component Analysis}

The relative importance of each character to explain the variation among the 30 A. hypogaea genotypes was assessed using the Principal Component Analysis (PCA). The first seven components explained $64.03 \%$ of the total variation among the 30 A. hypogaea accessions (Table 4). F1 separates accessions on the basis of three characters: branching pat- 
(a) Variables (axes F1 and F2: $\mathbf{3 0 , 4 0 \% )}$

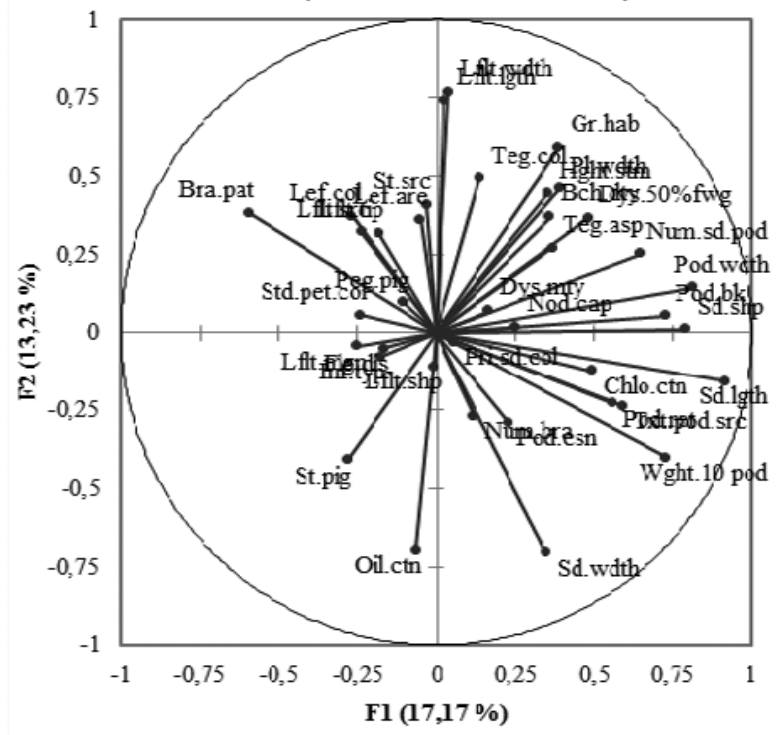

(b)

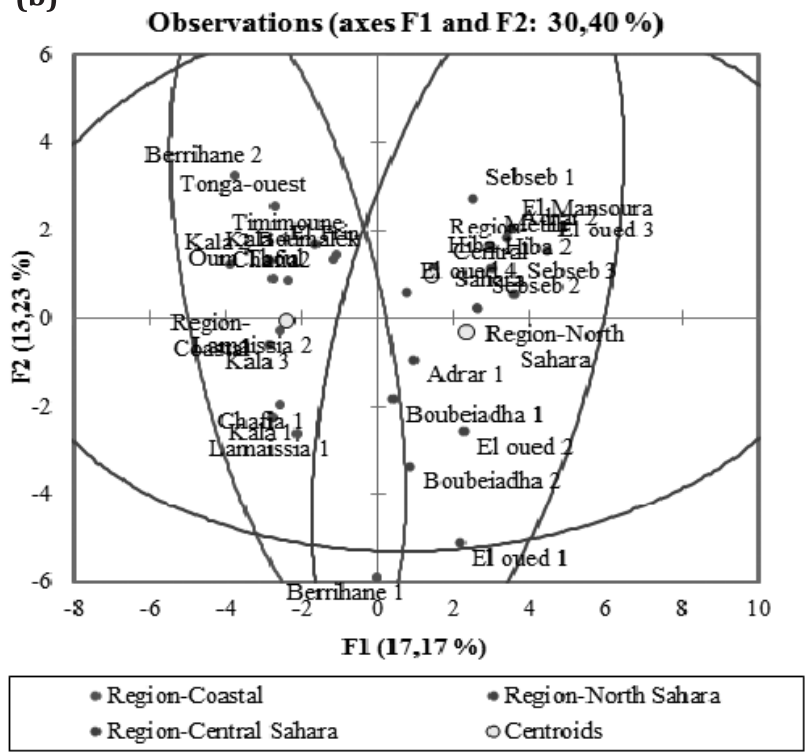

Fig 2. Principal Component Analysis of morphological characters:

(a) diagnostic biplot, (b) graph of observations

Рис. 2. Анализ морфологических признаков методом главных компонент:

(a) диагностическая диаграмма, (b) график наблюдений

Table 4. First seven principal components related to 38 characters studied in 30 Arachis hypogaea L. genotypes assessed

Таблица 4. Первые семь главных компонент для 38 изученных признаков у 30 проанализированных генотипов Arachis hypogaea L.

\begin{tabular}{|c|c|c|c|c|c|c|c|}
\hline & \multicolumn{7}{|c|}{ Principal component axis } \\
\hline & F1 & F2 & F3 & F4 & F5 & F6 & F7 \\
\hline Eigen value & 6.526 & 5.028 & 3.071 & 2.834 & 2.770 & 2.297 & 1.805 \\
\hline Variability (\%) & 17.174 & 13.231 & 8.081 & 7.459 & 7.291 & 6.044 & 4.751 \\
\hline Cumulative \% & 17.174 & 30.404 & 38.485 & 45.944 & 53.235 & 59.279 & 64.030 \\
\hline \multicolumn{8}{|c|}{ Eigen vector loadings } \\
\hline Gr.hab & 0.152 & 0.264 & 0.161 & -0.203 & 0.055 & 0.116 & -0.117 \\
\hline Bra.pat & -0.232 & 0.171 & 0.040 & -0.110 & 0.136 & 0.037 & -0.164 \\
\hline Num.bra & 0.046 & -0.120 & 0.333 & -0.060 & -0.210 & 0.093 & 0.147 \\
\hline Hght.stm & 0.138 & 0.198 & -0.061 & 0.080 & -0.027 & -0.186 & 0.058 \\
\hline Pl.wdth & 0.152 & 0.205 & 0.187 & 0.005 & -0.073 & 0.060 & -0.015 \\
\hline St.pig & -0.110 & -0.184 & 0.205 & 0.082 & 0.133 & 0.353 & 0.014 \\
\hline St.src & -0.012 & 0.183 & 0.100 & 0.322 & -0.131 & 0.186 & 0.036 \\
\hline Inf.typ & -0.068 & -0.034 & 0.350 & -0.193 & 0.024 & 0.115 & 0.125 \\
\hline Std.pet.col & $-0,095$ & 0.025 & 0.076 & -0.053 & -0.050 & 0.062 & 0.392 \\
\hline Peg.pig & -0.041 & 0.043 & -0.331 & -0.143 & -0.243 & 0.138 & 0.048 \\
\hline Flo.dis & -0.065 & -0.022 & -0.224 & 0.037 & -0.180 & 0.033 & -0.326 \\
\hline Lef.col & -0.105 & 0.166 & -0.182 & 0.024 & 0.174 & 0.002 & 0.190 \\
\hline Lflt.lgth & 0.008 & 0.331 & 0.152 & 0.056 & $-0,062$ & 0.201 & -0.019 \\
\hline Lflt.wdth & 0.015 & 0.343 & 0.079 & 0.064 & 0.081 & 0.057 & -0.155 \\
\hline
\end{tabular}


Table 4. The end

Таблица 4. Окончание

\begin{tabular}{|c|c|c|c|c|c|c|c|}
\hline & & & Pri & l compo & axis & & \\
\hline & F1 & F2 & F3 & F4 & F5 & F6 & F7 \\
\hline Lflt.shp & -0.002 & -0.048 & -0.193 & -0.028 & 0.061 & 0.402 & 0.172 \\
\hline Lflt.src & -0.093 & 0.144 & 0.165 & 0.356 & -0.042 & 0.144 & -0.168 \\
\hline Lflt.mgn & -0.099 & -0.018 & 0.120 & 0.279 & -0.228 & 0.024 & -0.181 \\
\hline Lflt.tip & -0.072 & 0.142 & -0.083 & -0.012 & -0.309 & 0.000 & -0.001 \\
\hline Dys.50\%fwg & 0.189 & 0.163 & 0.044 & -0.052 & 0.136 & -0.237 & 0.176 \\
\hline Dys.mty & 0.065 & 0.032 & -0.040 & 0.237 & 0.356 & -0.125 & 0.160 \\
\hline Chlo.ctn & 0.193 & -0.054 & 0.135 & -0.001 & -0.177 & -0.239 & 0.331 \\
\hline Bch.dty & 0.141 & 0.165 & 0.252 & 0.145 & -0.224 & -0.128 & 0.034 \\
\hline Nod.cap & 0.098 & 0.008 & -0.043 & 0.096 & -0.196 & 0.391 & 0.236 \\
\hline Lef.are & -0.019 & 0.162 & 0.021 & -0.140 & 0.395 & 0.227 & 0.019 \\
\hline Pod.csn & 0.089 & -0.130 & 0.132 & -0.281 & -0.225 & -0.033 & -0.087 \\
\hline Num.sd.pod & 0.253 & 0.112 & 0.096 & -0.206 & 0.112 & 0.045 & -0.014 \\
\hline Txt.pod.src & 0.231 & -0.105 & -0.110 & 0.122 & -0.012 & 0.026 & -0.324 \\
\hline Pod.bk & 0,284 & 0.024 & 0.059 & -0.168 & -0.125 & -0.051 & -0.082 \\
\hline Pod.ret & 0.219 & -0.103 & -0.052 & 0.048 & -0.039 & 0.300 & 0.015 \\
\hline Teg.asp & 0.145 & 0.120 & -0.184 & -0.182 & -0.114 & 0.052 & -0.101 \\
\hline Teg.col & 0.053 & 0.220 & 0.037 & 0.282 & 0.102 & -0.150 & 0.024 \\
\hline Pri.sd.col & 0.022 & -0.013 & -0.248 & 0.261 & -0.133 & -0.005 & 0.362 \\
\hline Oil.ctn & -0.025 & -0.311 & 0.271 & 0.024 & 0.105 & 0.031 & -0.030 \\
\hline Pod.wdth & 0.319 & 0.065 & -0.088 & 0.050 & -0.028 & 0.092 & -0.032 \\
\hline Wght.10 pod & 0.285 & -0.180 & 0.058 & 0.188 & 0.117 & 0.034 & -0.077 \\
\hline Sd.wdth & 0.136 & -0.315 & 0.032 & 0.234 & 0.117 & -0.044 & -0.101 \\
\hline Sd.lgth & 0.358 & -0.070 & -0.053 & 0.029 & 0.055 & 0.034 & 0.015 \\
\hline
\end{tabular}

tern, seed length, and pod width. Concurrently, F2 separates accessions on the basis of four characters: leaflet width and length, seed width, and oil content. According to the study by S. T. Katzamanidis et al. (2006), the major botanical accessions of A. hypogaea were previously classified on the basis of leaf and stem morphological differences. PCA results also revealed that leaflet size (length and width), seed shape and size, oil content, and branching pattern were the principal characters to discriminate between the examined $A$. hypogaea accessions. On the other hand, characters which have a low loading in the distinction of accessions were growth habit and leaf color for F1, and peg pigmentation and days to maturity for F2.

These results agree with those reported by P. Mahalakshmi et al. (2005), Idi Garba et al. (2015), and F. B. Neya et al. (2017). Regarding the regional distribution on the graph of observations (see Fig. 2, b), two main groups can be distinguished. The first group is composed of accessions from the coastal region, while the second one of those from the northern Saharan region. A third small group can be observed, in- corporating accessions from the central Sahara. The accessions from the coastal region were characterized by an alternate and sequential branching pattern, with flowers on the main stem, and small seed length and pod width. However, accessions from the northern Sahara presented an irregular branching pattern, without flowers on main stem. These accessions were characterized by high seed length and pod width. The third group consisted of accessions with more restricted distribution; accessions of this group were distinguished by a large size of leaflets and poor oil content.

4. Relationships among the studied accessions according to morphological characters

The relationships among the $30 \mathrm{~A}$. hypogaea accessions based on the studied morphological characters were assessed using cluster analysis. The typology of the dendrogram clustered the 30 accessions into two major groups identified as I and II, using a dissimilarity index limit of 157.64 (Fig. 3). The number of genotypes per cluster varied from 5 in cluster II to 13 in cluster I. Cluster I represented $43.33 \%$ of the studied population, including 12 accessions originated from Taref and 


\section{Dendrogram}

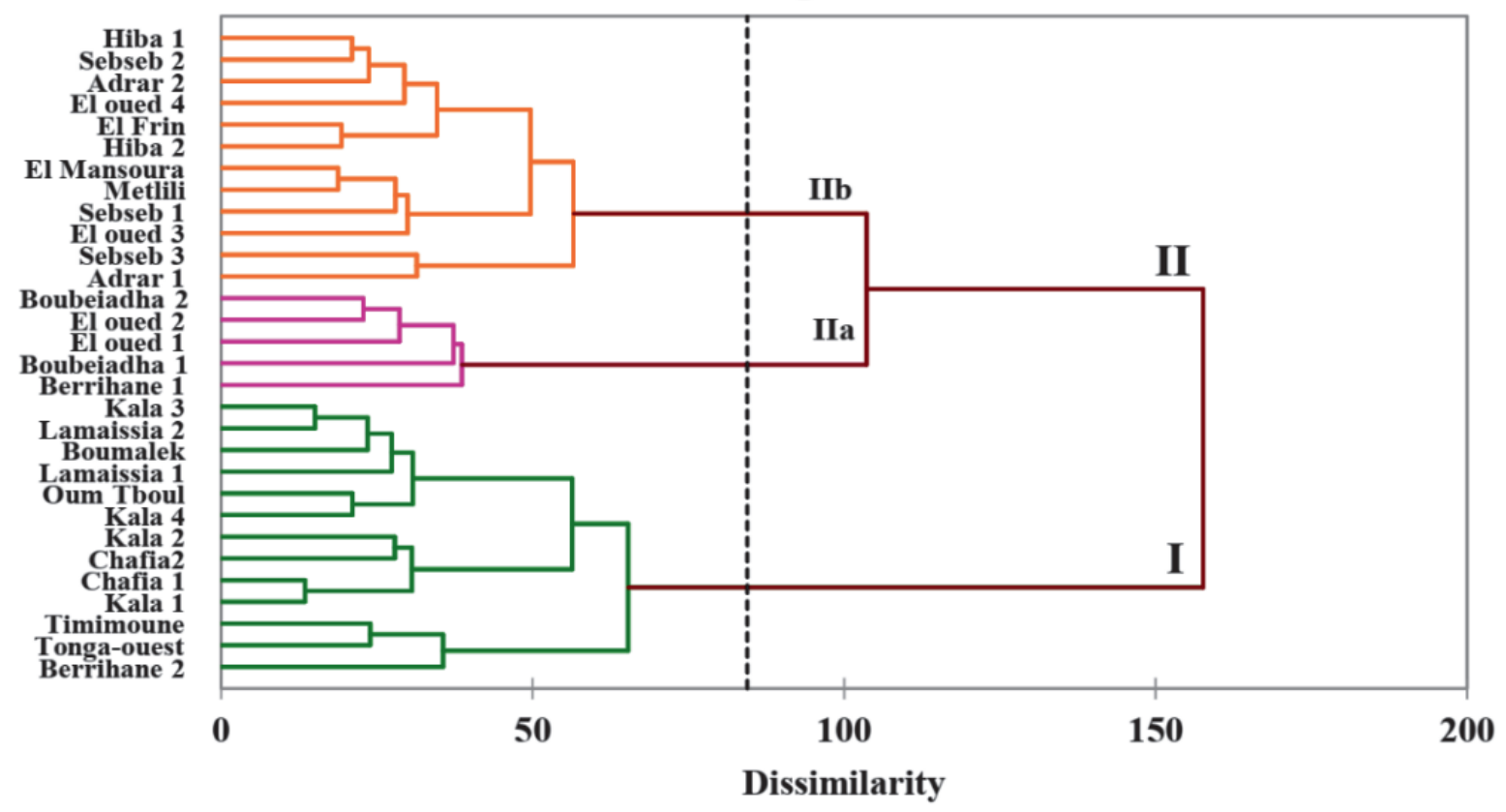

Fig. 3. Dendrogram based on the UPGMA method showing the relationships among the studied accessions Рис. 3. Дендрограмма на основе метода UPGMA, показывающая взаимосвязи среди изучаемых образцов

the only Timimoune accession from Adrar (see Table 1). This cluster was the most distinguished and the remoter one, supposedly because of the particular subhumid climate in the region of Taref.

The second major cluster (II) grouped most of the accessions originated from the northern and central Saharan regions and corresponded to $56.67 \%$ of the total studied population; this cluster was divided into two subclusters, IIa and IIb. The first subcluster, Ila, contains five accessions, four of which were from El Oued, whereas one accession originated from Taref (see Fig. 3 and Table 1), representing 16.66\% of the total studied population. Twelve accessions representing $40 \%$ of the total population, including those of different origin, namely Ghardaïa, Taref, El Oued, and Adrar, were distinctly grouped into subcluster IIb (see Fig. 3 and Table 1). This subcluster is the most heterogeneous among those identified; it is closer to subcluster IIa than the first one over a distance of 104.18. Except Berrihane 1 and El Frin, all accessions of the second cluster were cultivated in the Saharan climate. Clustering of these accessions in the same group can be explained by their adaptation to the specific climate of the Sahara.

\section{Analysis of gel electrophoresis}

In peanut, main seed storage proteins (about 87\%) are globulins comprising two major fractions: arachin and conarachin described by X. Q. Liang et al. (2006). Total proteins extracted from thirty peanut accessions were separated by onedimensional SDS-PAGE (Table 5), and the protein profiles revealed a few major quantitative and qualitative differences (Fig. 4); total bands distributed in the range of 250-6 kDa were scored. Electrophoresis profiles showed that different peanut accessions had different storage protein compositions generating 8 different genotypes presented in Figure 4.

The evaluation of band density using the Image Lab software version 5.1 (Biorad) revealed few differences among the studied accessions (data not shown). Most of the bands, including $4 \mathrm{a}, 3 \mathrm{~b}, 4 \mathrm{~b}, 1 \mathrm{c}, 2 \mathrm{c}, 3 \mathrm{c}, 4 \mathrm{c}, 1 \mathrm{~d}$ and $2 \mathrm{~d}$, presented variable density on the electrophoresis profile. This variation may be due to the adaptation of accessions to ecogeographic factors, soil richness in fertilizer like nitrogen, and the agronomic practices of the region (Chowdhury et al., 2015).

The number of protein bands present in each accession ranged from 23 to 30 bands. On the basis of the Rf value, the bands were grouped into four regions: region A (conarachin II), region B (acidic arachin), region C (basic arachin), and region $\mathrm{D}$ (conarachin I). No major qualitative variations were observed among the studied accessions in the arachin polypeptides (from $20 \mathrm{kDa}$ to $50 \mathrm{kDa}$ ). The second fraction of globulin, represented by conarachin polypeptides, was divided into two groups with different molecular weight $(>50 \mathrm{kDa}$ for conarachin II, and $<20 \mathrm{kDa}$ for conarachin I).

Regarding the electrophoresis profiles, the studied peanut accessions presented similar conarachin II profiles (region $\mathrm{A}$ ), characterized by a major protein of $61 \mathrm{kDa}$; this band corresponds to one of the important peanut allergens (Arah1).

In the electrophoresis profiles, 7 bands were identified in the region $\mathrm{D}$, among which the first and the second conarachin I protein subunits were highly polymorphic; they presented a molecular weight of $20.2 \mathrm{kDa}$ and $17.72 \mathrm{kDa}$, respectively, for most of the accessions, unlike 6 accessions of the collection from Taref and El Oued (Lamaissia 1, Lamaissia 2, Kala 3, El Oued 2, El Oued 3, and Boubeiadha 2) which missed these subunits. On the other hand, nine accessions, including, Chafia 1, Hiba 1, Sebseb 2, Metlili, El Mansoura, and others (see Fig. 4), presented these subunits with lower molecular weights (18.4 and 17.4, respectively). The accession Kala 4 had a specific profile and presented only the higher subunit (20.2 kDa).

6. Relationships among the studied accessions tested using storage protein separation

Cluster analysis of the accessions sorted the peanut population into three main groups at the distance of 56.8 (Fig. 5). Cluster I incorporated five accessions originated from Taref and Adrar, representing $16.66 \%$ of the total studied population. The second cluster represented $30 \%$ of the population and included 9 accessions of two different origins, Taref and Ghardaïa. 
Table 5. Protein profiling of Arachis hypogaea L. accessions Таблица 5. Профилирование белков у образцов Arachis hypogaea L.

\begin{tabular}{|c|c|c|c|c|}
\hline Band number & Band name & Region & $\begin{array}{c}\text { Molecular weight } \\
\text { (kDa) }\end{array}$ & Relative to front \\
\hline 1 & nl1 & \multirow{6}{*}{ NL } & 250.0 & 0.051 \\
\hline 2 & $\mathrm{nl} 2$ & & 238.9 & 0.067 \\
\hline 3 & nl3 & & 181.2 & 0.190 \\
\hline 4 & $\mathrm{nl} 4$ & & 174.7 & 0.212 \\
\hline 5 & nl5 & & 116.0 & 0.337 \\
\hline 6 & nl6 & & 110.5 & 0.353 \\
\hline 7 & a1 & \multirow{7}{*}{ A } & 103.4 & 0.362 \\
\hline 8 & a2 & & 94.6 & 0.394 \\
\hline 9 & a3 & & 85.7 & 0.425 \\
\hline 10 & $\mathrm{a} 4$ & & 78.2 & 0.441 \\
\hline 11 & a5 & & 68.1 & 0.476 \\
\hline 12 & a6 & & 61.0 & 0.525 \\
\hline 13 & a7 & & 57.2 & 0.542 \\
\hline 14 & b1 & \multirow{6}{*}{ B } & 54.6 & 0.583 \\
\hline 15 & b2 & & 50.3 & 0.610 \\
\hline 16 & b3 & & 44.5 & 0.629 \\
\hline 17 & b4 & & 43.2 & 0.648 \\
\hline 18 & b5 & & 40.5 & 0.673 \\
\hline 19 & b6 & & 37.2 & 0.702 \\
\hline 20 & $\mathrm{c} 1$ & \multirow{4}{*}{$\mathrm{C}$} & 35.0 & 0.735 \\
\hline 21 & c2 & & 32.4 & 0.771 \\
\hline 22 & c3 & & 29.5 & 0.783 \\
\hline 23 & c4 & & 28.0 & 0.799 \\
\hline \multirow{2}{*}{24} & \multirow{2}{*}{$\mathrm{d} 1$} & \multirow{9}{*}{ D } & 20.2 & 0.821 \\
\hline & & & 18.4 & 0.839 \\
\hline \multirow{2}{*}{25} & \multirow{2}{*}{$\mathrm{d} 2$} & & 17.72 & 0.850 \\
\hline & & & 17.40 & 0.841 \\
\hline 26 & d3 & & 14.74 & 0.873 \\
\hline 27 & $\mathrm{~d} 4$ & & 13.42 & 0.881 \\
\hline 28 & d5 & & 11.32 & 0.908 \\
\hline 29 & $\mathrm{~d} 6$ & & 8.851 & 0.932 \\
\hline 30 & $\mathrm{~d} 7$ & & 6 & 0.954 \\
\hline
\end{tabular}

Note: $\mathbf{N l}$ - non labelled

Примечание: $\mathrm{Nl}$ - немаркированный 

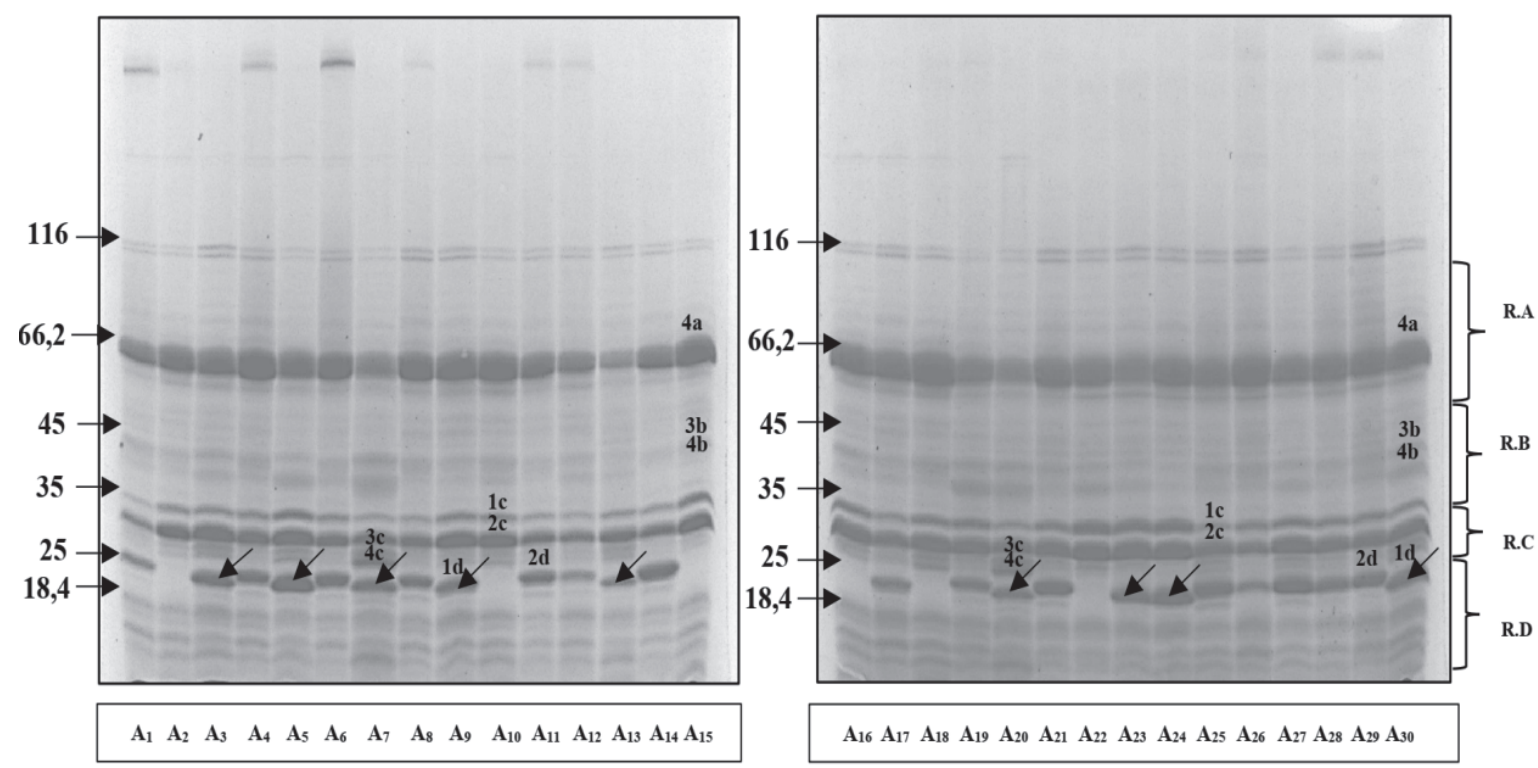

Fig. 4. Electrophoretic banding pattern generated by SDS-PAGE of Arachis hypogaea L. seed storage proteins: A1 - Berrihane 1, A2 - Lamaissia 1, A3 - Sebseb 2, A4 - Kala 1, A5 - Chafia 1, A6 - Boubeiadha 1, A7 - Hiba 1, A8 - Chafia 2, A9 - Hiba 2, A10 - Lamaissia 2, A11 - Adrar 1, A12 - Kala 2, A13 - Sebseb 1, A14 - El Oued 1, A15 - Kala 3, A16 - El Oued 2,

A17 - Adrar 2, A18 - Boubeiadha 2, A19 - El Frin, A20 - El Oued 4, A21 - Boumalek, A22 - El Oued 3, A23 - Metlili, A24 - Sebseb 3, A2 - Berrihane 2, A26 - Kala 4, A27 - Oum Tboul, A28 - Tonga-ouest, A29 - Timimoune, A30 - El Mansoura,

R.A - Region A, R.B - Region B, R.C - Region C, R.D - Region D, R.NL - Region non labelled

Рис. 4. Спектры электрофореза SDS-PAGE для запасных белков семян образцов Arachis hypogaea L.:

A1 - Berrihane 1, A2 - Lamaissia 1, A3 - Sebseb 2, A4 - Kala 1, A5 - Chafia 1, A6 - Boubeiadha 1, A7 - Hiba 1, A8 - Chafia 2, A9 - Hiba 2, A10 - Lamaissia 2, A11 - Adrar 1, A12 - Kala 2, A13 - Sebseb 1, A14 - El Oued 1, A15 - Kala 3, A16 - El Oued 2 ,

A17 - Adrar 2, A18 - Boubeiadha 2, A19 - El Frin, A20 - El Oued 4, A21 - Boumalek, A22 - El Oued 3, A23 - Metlili, A24 - Sebseb 3,A2 - Berrihane 2, A26 - Kala 4, A27 - Oum Tboul, A28 - Tonga-ouest, A29 - Timimoune, A30 - El Mansoura,

R.A - область A, R.B - область B, R.C - область C, R.D - область D, R.NL - немаркированная область

\section{Dendrogram}

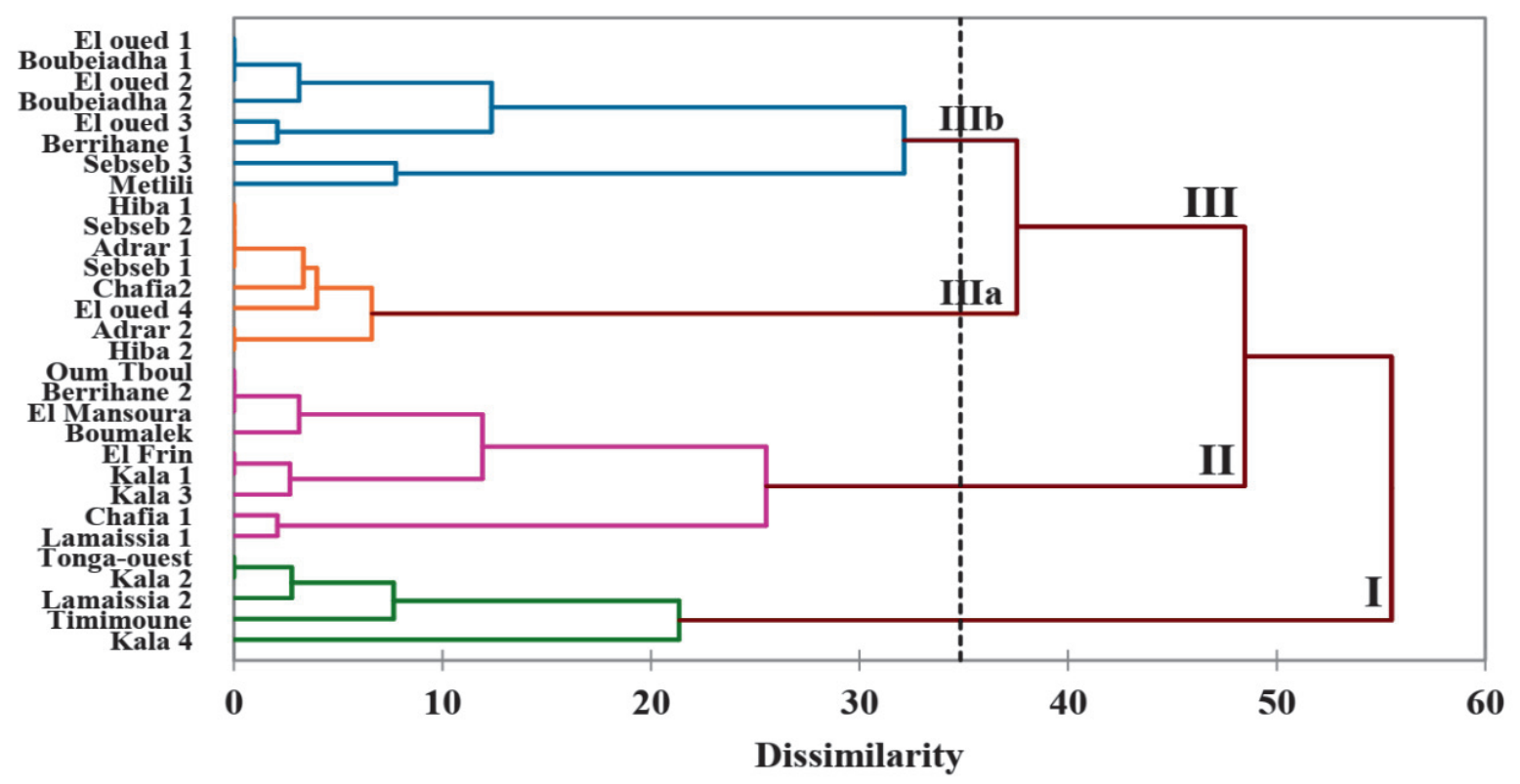

Fig. 5. Dendrogram generated using UPGMA cluster analysis based on storage seed proteins

Рис. 5. Дендрограмма, построенная с использованием кластерного анализа UPGMA на основе запасных белков семян 
The third cluster was composed of two subclusters (IIIa and IIIb). The first subcluster contained genotypes from different geographic regions: 8 accessions, including Hiba 2, Adrar 2, Chafia 2, Sebseb 1, and others. This subcluster described $26.66 \%$ of the whole population studied. Similarly, the second subcluster harbored 8 accessions of different geographic origin, like El Oued and Ghardaïa. These subclusters are the closest ones at a distance of 38.21 and were the most heterogeneous, harboring accessions of different origin. Many authors investigated seed storage proteins in the genus Arachis (Singh et al., 1991; Liang et al., 2006). M. Jamalomidi et al. (2015) analyzed eleven accessions of peanut germplasm by SDS PAGE and showed that the diversity exists for seed storage protein profiles, but the germplasm was not well classified into subspecies or varieties. In contrast, an attempt was made to characterize 35 peanut cultivars raised by different pedigrees, using total soluble seed proteins separated by SDSPAGE. Results showed a wide variation in the pattern of protein bands of the studied cultivars (Rao et al., 2013).

\section{Discussion}

To date, no study has assessed the variability of local peanut cultivars either at the morphological or at the molecular level. Hence, the current study is the first work where morphological characters were used to study the genetic diversity of A. hypogaea in Algeria. Diversity analysis using morphological characters is, therefore, very relevant for evaluation of genetic resources in the context of breeding programs.

The dendrogram describing the distances among accessions (see Fig. 2) revealed two distinguishes groups, clustering accessions originated from the coastal and Saharan regions. Generally, the studied Saharan accessions, including Ghardaïa, El Oued and Adrar, had high branching density and coarse texture of pod surface. This can be explained by the sunshine in the region and the type of sandy soil.

Branching in peanuts may produce a positive impact on yield, since the branches bear the leaves and also determine the solar radiation interception and utilization. In addition, the nodes of branches are potential sites for peg development and subsequent pod formation (Konlan et al., 2013). On the other hand, Saharan accessions, especially those of Ghardaïa, had the most significant height of main stems with the mean of $46.78 \mathrm{~cm}$, compared with coastal accessions which were characterized by smaller stems $(43.06 \mathrm{~cm})$. These observations agree with those of G. S. Zhao et al. (2007), where soil texture was the major factor affecting bean growth; they found that plants in loam and sand were significantly larger than in clay (Lu et al., 2010).

Cylindric seed shape was a specific trait of all Ghardaïa accessions; seeds and pods of these accessions were consequently the longest ones in the collection. This can increase the chance to get pods with many seeds and enhance the yield. In fact, the Ghardaiia and Adrar accessions more frequently contained 3 seeds in their pods than 2 seeds, unlike the Taref accessions which rarely had pods with 3 seeds. Knowing that soil texture is an important measure of potential soil productivity (Lu et al., 2010), Zhao and collaborators (2015) revealed that the number of seeds in peanuts was shown to be the largest in the loam, large in the sand, and the smallest in the clay.

Soil texture also affects the nodulation capacity of peanut. Indeed, sand soil was the poorest in organic matter, like phosphorus and nitrogen (Zhao et al., 2015). Therefore, peanut roots were able to form a nitrogen-fixing symbiosis with bacteria from the genus Rhizobium using nodules developed fre- quently on sand soils (Chevalier, 1934). Due to the soil texture, most of the Saharan accessions developed nodules on their root system. In contrast, all accessions originated from Taref produced glabrous roots. Except El Oued 03, the accessions originated from El Oued Province showed the best agronomic traits, thus being capable of improving peanut production because of their large seed size.

The El Oued accessions were the richest in oil content with an average of $48.31 \%$. In addition to that, these accessions mature in 80 to 100 days, and $50 \%$ of their flowers open between 20 to 45 days after sowing. These characteristics make the accessions that originated from El Oued Province the perfect target for producers due to their large seed size, short period of maturation, and high oil content.

According to A. Krapovickas and W. C. Gregory (1994), branching pattern and growth habit are the most distinguishing traits between A. hypogaea subspecies, such as A. hypogaea subsp. hypogaea and A. hypogaea subsp. fastigiata Waldron. In fact, subsp. hypogaea was characterized by an irregular branching pattern without flowers on the main stem and a procumbent growth habit. However, subsp. fastigiata was characterized by the absence of flowers on the main stem with an erect growth habit. Proceeding from the results shown on the graph, accessions of the collection can be divided into two groups: accessions of the coastal region belonging to subsp. fastigiata, and those of Saharan region belonging to subsp. hypogaea.

Judging by the results obtained by A. R. Singh et al. (2018), seed protein fractions can serve as an important marker to assess the variability among germplasm and also distinguish between the subspecies and varieties. These results support those of PCA and confirm the presence of the two subspecies of $A$. hypogaea in the studied collection. We deduce also that conarachin I protein subunits play a crucial role in the discrimination of $A$. hypogaea subspecies.

A high correlation was observed between the classification based on agro-morphological traits and the one based on storage proteins. In fact, the two classification criteria grouped the accessions originated from Taref and El Oued into distinct groups. However, a heterogeneous group was observed for the two classifications. Besides that, the characters used for both classifications can distinguish between the A. hypogaea subspecies. Nevertheless, it is suggested that the number of accessions should be increased for better understanding the genetic relationships.

\section{Conclusion}

Genetic improvement of crops depends on the availability of genetic resources and their diversity. We report here for the first time on the genetic diversity of the Algerian peanut population analyzed using agro-morphological traits. The results showed a high genetic variability regarding agro-morphological traits and seed storage proteins in the studied collection. This diversity could mainly be attributed to the diverse agroclimatic conditions in the country. Hence, the three groups defined on the dendrogram based on agro-morphological characters belonged to two A. hypogaea subspecies and may represent the required variability for a basic collection. Results also show that the accessions from El Oued presented the best agro-morphological traits and can be highly recommended for cultivation and production of peanut byproducts. With the aim to create an efficiency improvement program for Algerian peanut accessions, it is necessary to support this work with a genetic study to discriminate characters of agronomic importance. 
The authors are grateful to the Algerian Ministry of Higher Education and Scientific Research, the General Directorate of Scientific Research and Technological Development, Algeria, and the National Center for Biotechnology Research (CRBt) of Constantine.

Авторы благодарны Министерству высшего образования и научных исследований Алжира, Генеральному управлению научных исследований и технологического развития Алжира, Национальному центру биотехнологических исследований в г. Константин.

\section{References / Литература}

Chevalier A. Monographie de l'Arachide (Suite). Revue de botanique appliquée et d'agriculture coloniale. 1934;14(156):565-632. [in French] DOI: 10.3406/ jatba.1934.5400

Chowdhury F.N., Hossain D., Hosen M., Rahman S. Comparative study on chemical composition of five varieties of groundnut (Arachis hypogaea). World Journal of Agricultural Sciences. 2015;11(5):247-254. DOI: 10.5829/idosi. wjas.2015.11.5.1864

Descriptors for Groundnut. Rome: IBPGR; Patancheru: ICRISAT; 1992.

FAOSTAT. Food and Agriculture Organization of the United Nations. Food and agriculture data. FAO; 2020. Available from: http://faostat.fao.org/ [accessed Oct. 08, 2020].

Giuffrè A.M., Tellah S., Capocasale M., Zappia C., Latati M., Badiani M. et al. Seed oil from ten Algerian peanut landraces for edible use and biodiesel production. Journal of Oleo Science. 2016;65(1):9-20. DOI: 10.5650/jos.ess15199

Idi Garba N.M., Bakasso Y., Atta S., Mamane M.I., Adamou M., Hamidou F. et al. Evaluation of agro-morphological diversity of ground nut (Arachis hypogaea L.) in Niger. African Journal of Agricultural Research. 2015;10(5):334-344. DOI: 10.5897/AJAR2013.8364

Jamalomidi M., Gholami, M., Fatemeh J. Seed storage protein electrophoresis for identification of some groundnut (Arachis hypogaea L.) cultivars. International Research Journal of Applied and Basic Sciences. 2015;9(10):1718-1721.

Katzamanidis S.T., Stavropoulos N., Ipsilandis C.G. Classification and evaluation of Greek groundnut (Arachis hypogaea L.) using 17 main agronomic and quality traits. Pakistan Journal of Biological Science. 2006;9(6):1021-1027. DOI: 10.3923/pjbs.2006.1021.1027

Konlan S., Sarkodie-Addo J., Asare E., Kombiok M.J. Groundnut (Arachis hypogaea L.) varietal response to spacing in the Guinea Savanna agro-ecological zone of Ghana: Nodulation and nitrogen fixation. Agriculture and Biology Journal of North America. 2013;4(3):324-335. DOI: 0.5251/ abjna.2013.4.3.324.335

Krapovickas A., Gregory W.C. Taxonomía del género Arachis (Leguminosae). Bonplandia. 1994;8:1-186. [in Spanish]
Kushwah A., Gupta S., Sharma S.R., Kaur K. Study on genetic diversity in groundnut (study on genetic diversity in groundnut (Arachis hypogaea L.) using morphological markers. Supplement on Genetics and Plant Breeding. 2016;11(4):3105-3109.

Laemmli U.K. Cleavage of structural proteins during the assembly of the head of bacteriophage T4. Nature. 1970;227(5259):680-685. DOI: 10.1038/227680a0

Liang X.Q., Luo M., Holbrook C.C., Guo B.Z. Storage protein profiles in Spanish and runner market type peanuts and potential markers. BMC Plant Biology. 2006;6(1):24. DOI: $10.1186 / 1471-2229-6-24$

Lu H.D., Xue J.Q., Ma G.S., Hao Y.C., Zhang R.H., Ma X.F. Soil physical and chemical properties and root distribution in high yielding spring maize fields in Yulin, Shaanxi Province. Chinese Journal of Applied Ecology. 2010;21(4):895900.

Mahalakshmi P., Manivannan N., Muralidharan V. Genetic divergence of groundnut (Arachis hypogaea L.) germplasm. Legume Research - An International Journal. 2005;28(3):220-222.

Neya F.B., Koita K., Elise S., Zagre B.M.M., Nana A.T., Burow M.D. et al. Agro-morphological diversity of six peanut (Arachis hypogaea L.) breeding lines from three geographical areas. Journal of Experimental Agriculture International. 2017;19(5):1-12. DOI: 10.9734/ jeai/2017/37686

Rao P.S., Bharathi M., Reddy K.B. Identification of peanut (Arachis hypogaea L.) varieties through chemical tests and electrophoresis of soluble seed proteins. Legume Research. 2013;36(6):475-483.

Singh A., Raina S.N., Rajpal V.R., Singh A.K. Seed protein fraction electrophoresis in peanut (Arachis hypogaea L.) accessions and wild species. Physiology and Molecular Biology of Plants. 2018;24(3):465-481. DOI: 10.1007/ s12298-018-0521-8

Singh A.K., Sivaramakrishnan S., Mengesha M.H., Ramaiah C.D. Phylogenetic relations in section Arachis based on seed protein profile. Theoretical and Applied Genetics. 1991;82(5):593-597. DOI: 10.1007/BF00226795

Yamada T., Aibara S., Morita Y. Accumulation pattern of arachin and its subunits in maturation of groundnut seeds. Plant and Cell Physiology. 1980;21(8):1217-1226. DOI: 10.1093/oxfordjournals.pcp.a076120

Zaman M.A., Tuhina-Khatun M., Ullah M.Z., Moniruzzamn M., Alam K.H. Genetic variability and path analysis of groundnut (Arachis hypogaea L.). The Agriculturists. 2011;9(1-2):29-36. DOI: 10.3329/agric.v9i1-2.9476

Zhao C.X., Jia L.H., Wang Y.F., Wang M.L., McGiffen Jr M.E. Effects of different soil texture on peanut growth and development. Communications in Soil Science and Plant Analysis. 2015;46(18):2249-2257. DOI: 10.1080/00103624 .2015 .1059845

Zhao G.S., Li W.J., Sun Q. Effects of different soil texture on dry matter accumulation of Alfalfa. Xinjiang Agricultural Sciences. 2007;44(5):642-646. 
Прозрачность финансовой деятельности / The transparency of financial activities

Авторы не имеют финансовой заинтересованности в представленных материалах или методах.

The authors declare the absence of any financial interest in the materials or methods presented.

\section{Для цитирования / How to cite this article}

Джегхим Х., Беллиль И., Кхелифи Д. Генетическое разнообразие алжирской популяции арахиса, изученной с использованием морфологических маркеров и запасных белков семян. Труды по прикладнойботанике,генетикеи селекции. 2021;182(3):111-124. DOI: 10.30901/2227-8834-2021-3-111-124

Djeghim H., Bellil I., Khelifi D. Genetic diversity of the Algerian peanut population analyzed using morphological markers and seed storage proteins. Proceedings on Applied Botany, Genetics and Breeding. 2021;182(3):111-124.DOI:10.30901/2227-8834-2021-3 111-124
Авторы благодарят рецензентов за их вклад в экспертную оценку этой работы / The authors thank the reviewers for their contribution to the peer review of this work

\section{Дополнительная информация / Additional information}

Полные данные этой статьи доступны / Extended data is available for this paper at https://doi.org/10.30901/2227-8834-2021-3-111-124

Мнение журнала нейтрально к изложенным материалам, авторам и их месту работы / The journal's opinion is neutral to the presented materials, the authors, and their employer

Авторы одобрили рукопись / The authors approved the manuscript

Конфликт интересов отсутствует / No conflict of interest

ORCID

Djeghim H. https://orcid.org/0000-0001-7913-6346

Bellil I https://orcid org/0000-0003-2718-3102

Khelifi D. https://orcid.org/0000-0001-8139-5064 